\title{
Artritis reumatoide temprana
}

\author{
Loreto Massardo
}

\section{Early rheumatoid arthritis}

This article reviews some key issues of early rheumatoid arthritis such as the difficulties to recognize this condition during the first months after onset. Therefore, three reference diagnostic criteria have been proposed for any patient presenting with more than three simultaneously inflammed joints, involvement of metacarpophalangeal or proximal interphalangeal joints and morning stiffness lasting more than 30 minutes. Antibodies to cyclic citrullinated peptides are new markers that can be used for diagnosis. The immediate treatment during the "opportunity window" at the onset of inflammation may avoid the erosive joint damage. The use of synthetic or biological disease modifying medications, specially tumor necrosis factor alpha antagonists, also contribute to this purpose. Primary care physicians should be aware of the early signs of the disease to provide an adequate treatment and referral to specialists (Rev Méd Chile 2008; 136: 1468-75).

(Key words: Arthritis, rheumatoid; Early diagnosis; Tumor necrosis factor-alpha)

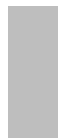

Recibido el 3 de diciembre, 2007. Aceptado el 7 de abril, 2008. Actualizado el 2 de octubre, 2008.

Departamento de Inmunología Clínica y Reumatología, Facultad de Medicina, Pontificia Universidad Católica de Chile. Santiago, Chile.

\section{ARTRITIS REUMATOIDE TEMPRANA}

L a artritis reumatoide (AR) es una enfermedad inflamatoria crónica sistémica cuya expresión clínica más importante es una poliartritis erosiva junto con pruebas serológicas de autorreactividad. Afecta entre $0,4 \%$ y $1 \%$ de la población de América Latina ${ }^{1}$ y es mucho más común en mujeres que en hombres, con una relación de 68:1 en esta región ${ }^{2}$. Se caracteriza por el dolor crónico y destrucción articular, por mortalidad prematura y por presentar un riesgo de invalidez elevado con altos costos para el enfermo y la sociedad. Sin tratamiento son esperables la incapacidad progresiva y la invalidez al cabo de un

Correspondencia a: Dra. Loreto Massardo. Marcoleta 352. Teléfono: 6397750. Fax: 6324937.

E mail: massardo@med.puc.cl lapso que va de pocos meses a 20 años de evolución, dependiendo de la presencia de factores pronósticos ${ }^{3-5}$. Muy importante para quienes la padecen es la pérdida de la capacidad laboral, en especial si se trata de trabajadores manuales, pues muchos dejan de percibir ingresos ya en los dos primeros años de enfermedad ${ }^{6,7}$.

Esta situación contrasta con la afirmación realizada en 1995 por investigadores ingleses que postularon que la AR es en la época actual la causa más común de incapacidad potencialmente tratable en el mundo occidental ${ }^{8}$. Esto parece ser cierto siempre que el tratamiento se inicie en etapas tempranas de la enfermedad y con terapias eficaces cuya meta sea obtener su remisión. Lo anterior implica un cambio en el paradigma de la terapia en AR hacia una aplicación inmediata de las nuevas terapias efectivas o de esquemas de combinación de estas drogas. El resultado del tratamiento comenzado en etapas más tardías o con daño establecido no será exitoso en un número impor- 
tante de pacientes, aun incluyendo terapias biológicas con anticuerpos o receptores solubles, bloqueadores del factor de necrosis tumoral (FNT) alfa, que es una muy potente citoquina proinflamatoria ${ }^{9-11}$. El objetivo de esta revisión es destacar uno de los aspectos más novedosos que han sido desarrollados en los últimos años en el tratamiento medicamentoso de la AR.

\section{DEFINICIÓN DE AR TEMPRANA}

La definición de cuándo considerar que estamos frente a una AR de comienzo reciente ha tenido variaciones que han dependido de los distintos autores que han trabajado en este tema. Algunos fijan los límites de la AR temprana hasta en tres años a partir del inicio de los síntomas. Sin embargo, dado que muchas veces el daño ya se está produciendo en ese período ${ }^{12,13}$, tiene más sentido si se considera como AR temprana el primer año transcurrido desde el inicio de los síntomas. Otros autores han acuñado el término de AR muy temprana, incluyendo la artritis de pocas semanas a pocos meses de evolución ${ }^{14}$. Este punto no es menor, puesto que si bien el diagnóstico de AR sigue siendo clínico, en la práctica médica habitual se establece al reunir el cumplimiento de 4 de los 7 criterios de la clasificación diagnóstica del American College of Rheumatology (ACR) en 198715, lo que ocurre luego de un tiempo de evolución de esta enferme- dad crónica, que en muchos de los casos puede ser prolongado. Por esto, autores holandeses han propuesto criterios de diagnóstico de AR temprana de modo de predecir el curso hacia una artritis persistente erosiva ${ }^{16}$. Los criterios que considera el modelo holandés se muestran en la Tabla 1, e incluye la presencia de anticuerpos antipéptidos cíclicos citrulinados (anti CCP) en el suero.

Como es conocido, la autoinmunidad es un fenómeno que subyace en la patogenia de la $A R$, de modo que los individuos que la padecen presentan anticuerpos contra una variedad de antígenos, entre los que se encuentran los dirigidos contra los aminoácidos citrulinados. Estos se generan por acción post translacional de la enzima peptidilarginina deiminasa, la que deiminiza los residuos de arginina transformándolos en citrulina. Los anti CCP fueron descritos en 1998 por Schellekens $^{17}$, y hasta ahora se ha identificado como autoantígenos citrulinados a la filagrina, al fibrinógeno y a la fibrina. Los anti CCP constituyen un nuevo marcador de AR que se puede detectar años antes del comienzo de la enfermedad, parecen ser muy específicos de AR, permanecen estables en el tiempo y se asocian con una enfermedad agresiva y erosiva. Así, representan una ventaja por sobre el factor reumatoide, pues tienen una mayor especificidad para el diagnóstico de AR, alrededor de 90\% y son una gran ayuda para la pesquisa de pacientes con AR temprana, especialmente aquellos pacientes que aún no cumplen criterios diagnósticos ACR 87 para la enfermedad ${ }^{18}$.

\section{Tabla 1. M odelo holandés para predecir una artritis persistente (erosiva)*}

Duración de síntomas a la primera visita de 12 semanas

Rigidez matutina por $60 \mathrm{~min}$

Artritis en más de 3 articulaciones

Dolor a la compresión bilateral de las articulaciones metatarsofalángicas

Anticuerpos antipéptidos cíclicos citrulinados positivos

Erosiones visibles en las radiografías de manos y pies

*Referencia 16. 


\section{EL CONCEPTO DE «ENTANA DE OPORTUNIDAD»}

Este concepto se refiere a un período muy breve al inicio de la artritis en el que se establecería la progresión radiológica a futuro y se basa en estudios en los que una intervención de inducción terapéutica intensa al inicio de la AR pudo reprogramar» la tasa de progresión de daño radiográfico ${ }^{19}$ mientras que un retraso de 3 a 9 meses en comenzar fármacos antirreumáticos modificadores de enfermedad (FARMEs) impactó negativamente en el desenlace radiográfico al cabo de 2 años ${ }^{20,21}$. El concepto de ventana de oportunidad postula que el uso de las terapias más potentes actualmente disponibles serán más efectivas cuanto menor sea la duración de la $\mathrm{AR}^{22,23}$, lo que eventualmente conducirá a una menor probabilidad de incapacidad. Esto ha abierto la esperanza de que los enfermos en un futuro no requerirán reemplazos articulares o cirugías ortopédicas y tendrán una menor mortalidad por ateroesclemosis prematura. Desde el año 2000 ha habido un cambio notable hacia la mejoría en el pronóstico de la AR para aquellos pacientes que reciben terapias durante la ventana de oportunidad ${ }^{24}$. Estas nuevas ideas plantean la necesidad que estos enfermos sean vistos por reumatólogos lo antes posible y no con un promedio de evolución de la enfermedad de 4 años, que fue lo que encontramos en nuestro estudio de $1995^{6}$.

\section{CRITERIOS DE DERIVACIÓN INMEDIATA A ESPECIALISTA}

La recomendación entonces para los médicos y el personal de salud que trabaja en atención primaria, para aquellos que por su especialidad reciben consultas por síntomas articulares y para quienes atienden preferentemente a mujeres es: Identificar a los pacientes eventuales con AR o con sospecha de AR. Es decir que presenten cualquiera de las siguientes manifestaciones que sustentan el diagnóstico probable de AR temprana, y que se conocen como los criterios de referencia de AR temprana de Emery ${ }^{25}$. Estos son:

1. Más de tres articulaciones inflamadas (hinchadas y dolorosas) simultáneamente.

2. Articulaciones metacarpofalángicas o metatarsofalángicas afectadas.

3. Rigidez matutina mayor a $30 \mathrm{~min}$.
CONCEPTO DE ARTRITIS INDIFERENCIADA, AR PROBABLE O EN POTENCIA

Un problema de la instalación de terapia en pacientes muy al inicio de los síntomas y que no cumplan con criterios diagnósticos de AR es el riesgo de tratar en exceso individuos con terapias que poseen efectos adversos potencialmente graves. Es posible que alrededor de la mitad de los enfermos que consultan en las clínicas de sartritis muy reciente»no evolucionarán a $\mathrm{AR}$ sino que al cabo de un año de observación habrán mejorado o presentarán otras artropatías incluyendo artritis virales, artritis por cristales de pirofosfatos, artrosis, artropatía diabética, espondiloartropatías o enfermedades del tejido conectivo. El estudio PROMPT probó la eficacia de tratar con metotrexate a 55 pacientes de un grupo de 110 pacientes que consultaron por artritis indiferenciada 0 que cumplían con los antiguos criterios de artritis probable (5 semanas o menos de estos síntomas: rigidez matutina, dolor en la movilización 0 en al menos una articulación, hinchazón de una articulación, hinchazón de al menos alguna otra articulación, hinchazón simétrica). Al cabo de un año 51 enfermos (46\% del total) había desamollado AR. Es interesante destacar en ese estudio, que 22 (81\%) de los 27 pacientes portadores de antiCCP desarrollaron AR en ese período ${ }^{26}$.

\section{FACTORES PRONÓSTICOS}

El objetivo de la terapia de la AR es el control riguroso de la inflamación para que al detener así el avance de la enfermedad, se pueda prevenir la erosión articular y que sobrevenga la incapacidad posterior. Para esto se necesita establecer un diagnóstico temprano en el curso de la $\mathrm{AR}$, identificar a los pacientes con más probabilidad de cursar con una forma rápidamente progresiva e iniciar una terapia más intensa en estos pacientes con mal pronóstico. Los factores de peor pronóstico reconocidos en AR temprana se presentan en la Tabla 2, a los que se ha agregado el tabaquis$\mathrm{mo}^{27,28}$. La relación entre tabaco y artritis es compleja y controvertida; se postula que existińa una relación entre fumar con una mayor frecuencia de autoanticuerpos antiCCP y un riesgo elevado de desarmollar $\mathrm{AR}$; y que esta asociación senáa mucho más fuerte en los portadores de factores genéticos para $\mathrm{AR}^{29}$. Por otro lado el papel del marcador genético de la $\mathrm{AR}$ 
Tabla 2. Factores de peor pronóstico en AR temprana

\author{
Gran número de articulaciones activas \\ Velocidad horaria de sedimentación o proteína C reactiva altas a la partida \\ Factor reumatoide positivo y anticuerpos antipéptidos cíclicos citrulinados positivos \\ Desarrollo de erosiones radiológicas tempranamente \\ Gran incapacidad funcional a la presentación \\ Pocos años de educación formal \\ Condiciones socioeconómicas adversas \\ Tabaquismo
}

denominado «epitopo compartido», situado en el cromosoma 6 en el complejo del sistema de histocompatiblidad HLA clase II, se asocia no sólo con desamollar la enfermedad sino también con formas más graves con manifestaciones extraarticulares y más erosivas de AR en población caucásica, también estas relaciones se encuentran presentes aunque en una menor proporción en pacientes chilenos con AR establecida $^{30}$. Los estudios de los marcadores inmunogenéticos en AR de reciente inicio están en marcha.

\section{IMÁGENES}

La presencia de erosiones en la radiología convencional de pies y manos suele ser tardía para los efectos de diagnóstico y prevención de daño en AR. Quizá si su verdadero valor esté en la exclusión de artritis por pirofosfatos en pacientes con AR muy reciente. En cambio, el ultrasonido y la resonancia magnética de extremidades tienen utilidad en la detección de sinovitis y de erosiones no visibles en la radiología 31,32 .

\section{TERAPIA}

Como ya se ha señalado, la AR debiera ser tratada lo antes posible para impedir daño irreversible. En este sentido, la Liga Europea EULAR desarrolló guías de terapia para AR temprana ${ }^{33}$. En ellas establece 12 recomendaciones entre las que destacan que los pacientes con artritis en una articulación deben ser examinados por un reumatólogo dentro de 6 semanas del inicio de síntomas, comenzar terapia con antiinflamatorios no esteroidales, esteroides (orales, intramusculares o intraarticulares) por un corto lapso y el uso del metotrexate como droga ancla. También recomienda la utilización del monitoreo clínico con empleo de conteos de articulaciones inflamadas, como el índice de actividad de 28 articulaciones (Disease activity score DAS28 ${ }^{34}$, uso de cuestionarios de capacidad funcional como es el cuestionario de autoevaluación de salud $4 \mathrm{HAQ} \aleph^{35}$ y de marcadores de inflamación como la proteína $\mathrm{C}$ reactiva, cada 1 a 3 meses. Recomienda también el empleo de técnicas de imágenes para monitorización de daño articular, cada 6 meses o anual. Además sugiere realizar los ajustes con terapias más potentes si no se ha logrado un control satisfactorio de la inflamación o el daño.

La guía nacional de la Sociedad Chilena de Reumatología ${ }^{36}$ y la del «American College of Rheumatology» 37 recomiendan que el tratamiento de un paciente con AR comience dentro de los tres primeros meses del diagnóstico, para lo que muchos reumatólogos seleccionarán al metotrexa- 
te como el fármaco antirreumático modificador de enfermedad (FARME) de primera línea en AR activa, pues es seguro y barato. Sin embargo se estima que 15\% de los enfermos no responden con metotrexate y $60 \%$ presenta una respuesta parcial ${ }^{38}$. Para estos pacientes se encuentran disponibles otros FARMEs con capacidad potencial de disminuir la progresión radiológica: leflunomida, azulfidine, ciclosporina $\mathrm{A}$, sales de oro inyectables ${ }^{39}$, combinaciones entre ellos o con fármacos biológicos ${ }^{11}$. Un problema real que hace que los reumatólogos tengamos pocas oportunidades de utilizar los agentes biológicos es el precio que sobrepasa ampliamente al del metotrexate. Uno de los temas más candentes en reumatología es establecer si este mayor valor es costo-efectivo. Una experiencia nacional en efectividad y seguridad se está formando en aquellos centros que participan como colaboradores en estudios internacionales de investigación clínica con fármacos y medicamentos biológicos en artritis reumatoide ${ }^{40}$.

Las opciones de terapia en AR inicial en la actualidad son variadas, con esquemas terapéuticos que no usan esteroides o los emplean en dosis altas o bajas, uso de FARMES solos o combinaciones de FARMES, FARMES con fármacos biológicos y esquemas en adición o en sustracción de medicamentos (Tabla 3). El estudio Best ${ }^{41}$ ejemplifica esta variedad al comparar 4 esquemas de terapia en que las decisiones terapéuticas se ajustaron según criterio de remisión del índice DAS. Si bien todos los esquemas de remisión fueron exitosos, se comportaron mejor los que incluían drogas bloqueadores del FNT alfa al obtener más rápido una respuesta clínica más potente y más duradera, llegando en algunos casos a suspender el agente biológico estudiado al cabo de 1 año. Se destaca que se observó un daño radiológico menor en aquellos pacientes que recibieron terapia con inhibidores del FNT alfa.

La neutralización de algunas de las citoquinas involucradas en la patogénesis de las enfermedades inflamatorias es una modalidad terapéutica establecida en la actualidad. El primer ejemplo fue la inhibición del factor de necrosis tumoral alfa (por infliximab, etanercept y adalimumab) en la terapia de la AR y de otras enfermedades autoinmunes inflamatorias. Por otra parte el desarrollo de más blancos terapéuticos potenciales se encuentra en plena evolución, como son los que ofrecen la compleja red de interleuquinas (IL) (IL-1, IL-6, IL15, IL-17) y varias adipoquinas; estrategias contra blancos celulares que incluyen anticuerpos contra CD20, anti BLyS y anti APRL, las que agotan o inhiben los linfocitos B; o estrategias moduladoras de la co-estimulación de células $\mathrm{T}^{42}$. En la Tabla 4 se muestran algunos fármacos biológicos en uso en AR. El bloqueo del FNT alfa en la práctica clínica se asocia con reducción de la actividad inflamatoria y daño, y con mejoría de la calidad de vida ${ }^{11,43}$. Los Drs. Scott y Kinsgley ${ }^{44}$ reunieron los datos de seis mil pacientes con AR que participaron en varios estudios aleatorios y comparativos de metotrexate versus anti TNF alfa combinado con metotrexate según criterios de respuesta del American College of Rheumatology ACR7045. En aquellos con AR temprana (menos de 3 años) la tasa de respuesta a metotrexate fue de $19 \%$ a $21 \%$ y la respuesta a bloqueadores del FNT alfa combinado con metotrexate de $33 \%$ a $40 \%$. En cambio en pacientes con AR establecida (más de 3 años) las tasas de

Tabla 3. Conceptos en el tratamiento de la AR temprana

\begin{tabular}{|ll|}
\hline $\begin{array}{l}\text { Tratar pronto } \\
\text { e intensivamente }\end{array}$ & - $\begin{array}{l}\text { Metotrexate es la primera elección en AR } \\
\text { oral o parenteral semanal }\end{array}$ \\
\hline $\begin{array}{l}\text { Usar combinaciones } \\
\text { de medicamentos }\end{array}$ & - $\begin{array}{l}\text { Combinaciones de fármacos antirreumáticos modificadores } \\
\text { de enfermedad sintéticos* }\end{array}$ \\
\hline Objetivo & - Metotrexate más un agente biológico \\
\hline
\end{tabular}

*Metotrexate, cloroquina, hidroxicloroquina, leflunomida, azulfidina, ciclosporina A, sales de oro inyectables 


\section{Tabla 4. Ejemplos de terapias biológicas en el tratamiento de la artritis reumatoide}

\begin{tabular}{|c|c|}
\hline & $\begin{array}{l}\text { Comentarios: } \\
\text { - Son más efectivas combinadas con metotrexate } \\
\text { - } \quad \text { Uso reservado para casos de pacientes con insuficiente respuesta o intolerancia a FARMEs* } \\
\text { - Se plantea el uso por } 1 \text { año como inductores de remisión en pacientes con AR temprana. }\end{array}$ \\
\hline & $\begin{array}{l}\text { Inhibidores del factor de necrosis tumoral alfa: } \\
\text { Infliximab, adalimumab, etanercept } \\
\text { Nuevos: certozulimab pegol, golimumab }\end{array}$ \\
\hline & $\begin{array}{l}\text { Terapia moduladora de la activación de células } \mathrm{T} \text { : } \\
\text { Abatacept }\end{array}$ \\
\hline & $\begin{array}{l}\text { Terapia anticélulas B : } \\
\text { Rituximab } \\
\text { Nuevos: ocrelizumab, atacicept }\end{array}$ \\
\hline & $\begin{array}{l}\text { Terapia antirreceptor de interleuquina } 6 \text { : } \\
\text { Tocilizumab }\end{array}$ \\
\hline & $\begin{array}{l}\text { Inhibición de vías de traducción de señales: } \\
\text { Vías: NF } \kappa \text { B, MAP kinasas, JAK-STAT }\end{array}$ \\
\hline
\end{tabular}

*FARMEs: fármacos antirreumáticos modificadores de enfermedad sintéticos: metotrexate, cloroquina, hidroxicloroquina, leflunomida, azulfidina, ciclosporina A, sales de oro inyectables.

respuesta fueron a metotrexate: $5 \%$ y a inhibidores de anti TNF alfa más metotrexate entre $10 \%$ y $27 \%$. Un metaanálisis de 29 estudios aleatorios y controlados de los tres antiFNT que reunió 9.939 sujetos con AR, señaló que el número necesario para tratar (NNT) con 70\% de mejoría en criterios ACR70 es 7,7 (95\% intervalo de confianza: 8-11) en AR establecida. En cambio en pacientes con AR temprana el NNT fue: 5,6 (95\% IC: 3,8-10). El número necesario para causar daño ( number necessary to harm» 0 $\mathrm{NNH}$ ) como infecciones (entre ellas la reactivación de tuberculosis e infecciones por patógenos comunes) fue: 25 (95\% IC: 16-100) en AR temprana. En AR establecida el $\mathrm{NNH}$ no se reportó por ser información comencial reservada ${ }^{46}$.

Otro aspecto importante a destacar es que los esteroides utilizados en dosis bajas y dados al inicio de la AR tienen demostradamente menos efectos adversos de lo que se pensaba. Su uso otorga alivio a los pacientes con un buen perfil de seguridad 47 , además de disminuir la progresión radiológica varios años después de haber sido suspendidos ${ }^{48}$.

A pesar de los avances recientes, las causas de la AR siguen siendo desconocidas y aún queda un grupo de enfermos refractarios a agentes biológicos. Se requiere urgentemente de más investiga- ción clínica y básica que permitan comprender la etiopatogenia de esta enfermedad y desarrollar nuevas opciones terapéuticas.

En nuestro país la AR ha ingresado entre las 56 patologías con garantías explícitas en salud (G.E.S.) por su considerable «carga de enfermedad». Como la AR en sus inicios suele ser difícil de reconocer y es complejo decidir quiénes necesitan tratamientos más potentes desde el comienzo, resulta relevante que el diagnóstico se haga lo más tempranamente posible, lo que se puede lograr mediante una mejor información al público y a los médicos que trabajan en atención primaria. Se necesitaría además establecer una mejor organización de la forma de prestar atención de salud a esta población, de modo de romper las brechas que hoy dificultan el acceso a medicamentos y a atención por especialistas. Es deseable que en la atención a estos pacientes se utilice una evaluación con índices objetivos, cuantitativos y periódicos en el curso de la enfermedad. Asimismo que se cumpla con el acceso a tratamientos eficaces, lo que incluye disponibilidad de agentes biológicos en casos seleccionados, para poder instaurar terapia efectiva en un momento en el que aún se puede influir en el desenlace de la enfermedad. 


\section{REFERENCIAS}

1. Cardiel MH. First Latin American position paper on the pharmacological treatment of rheumatoid arthritis. Rheumatology (Oxford). 2006; 45 Suppl 2: ii7ii22.

2. Pons-Estel B, Gladar. Cohorte Latino Americana de pacientes con artritis reumatoide de reciente comienzo (ARRC). Grupo Latino Americano de Estudio De Artritis Reumatoide. Reumatología 2005; 21: 165.

3. Wolfe F, Zwiшch SH. The long-term outcomes of rheumatoid arthritis: a 23-year prospective, longitudinal study of total joint replacement and its predictors in 1,600 patients with rheumatoid arthritis. Arthritis Rheum 1998; 41: 1072-82.

4. LeE DM, WeinBLatT ME. Rheumatoid arthritis. Lancet 2001; 358: 903-11.

5. LIPSKY P. Rheumatoid arthritis. In: Kasper D, Braunwald E, Fauci A, Hauser S, Longo D, Jameson J, eds. Harrisons. Principles of Internal Medicine. $16^{\text {th }}$ ed. Vol. 1. New York: McGraw-Hill; 2005; 1968-77.

6. Massardo L, Aguirre V, Garcia ME, Cervilia V, Nicovani S, Gonzalez A ET AL. Clinical expression of rheumatoid arthritis in Chilean patients. Semin Arthritis Rheum 1995; 25: 203-13.

7. Young A, Dixey J, Cox N, Davies P, Devin J, Emery P et AL. How does functional disability in early rheumatoid arthritis (RA) affect patients and their lives? Results of 5 years of follow-up in 732 patients from the Early RA Study (ERAS). Rheumatology (Oxford) 2000; 39: 603-11.

8. EMERY P, SALMON M. Early rheumatoid arthritis: time to aim for remission? Ann Rheum Dis 1995; 54: 944-7.

9. ScotT DL. Prognostic factors in early rheumatoid arthritis. Rheumatology (Oxford) 2000; 39 Suppl 1: 24-9.

10. FIRESTEIN GS. Evolving concepts of rheumatoid arthritis. Nature 2003; 423: 356-61.

11. Olsen NJ, Stein CM. New drugs for rheumatoid arthritis. N Engl J Med 2004; 350: 2167-79.

12. Bukhari M, Harrison B, Lunt M, Scott DG, Symmons DP, SILMAN AJ. Time to first occurrence of erosions in inflammatory polyarthritis: results from a prospective community-based study. Arthritis Rheum 2001; 44: 1248-53.

13. BreEdveld FC, KaLden JR. Appropriate and effective management of rheumatoid arthritis. Ann Rheum Dis 2004; 63: 627-33.

14. Machold KP, Eberl G, LeEb BF, NeLl V, Windisch B, SMOLEN JS. Early arthritis therapy: rationale and current approach. J Rheumatol Suppl 1998; 53: 13-9.

15. Arnett FC, Edworthy SM, Bloch DA, Mcshane DJ, FRIES JF, COOPER NS ET AL. The American Rheumatism Association 1987 revised criteria for the classification of rheumatoid arthritis. Arthritis Rheum 1988; 31: 315-24.
16. Visser H, Le Cessie S, Vos K, Breedveld FC, Hazes JM. How to diagnose rheumatoid arthritis early: a prediction model for persistent (erosive) arthritis. Arthritis Rheum 2002; 46: 357-65.

17. Schelemens GA, De Jong BA, Van Den Hoogen FH, Van De Putte LB, Van Venrooij WJ. Citrulline is an essential constituent of antigenic determinants recognized by rheumatoid arthritis-specific autoantibodies. J Clin Invest 1998; 101: 273-81.

18. Steiner G. Autoantibodies in rheumatoid arthritis. In: Hochberg MC, Silman A, Smolen JS, Weinsblat ME, Weisman MH, eds. Rheumatology Third ed. Vol. 1. Mosby; 2003; 833-41.

19. Boers M, Verhoeven AC, Markusse HM, Van De Laar MA, Westhovens R, Van Denderen JC et al. Randomized comparison of combined step-down prednisolone, methotrexate and sulphasalazine with sulphasalazine alone in early rheumatoid arthritis. Lancet 1997; 350: 309-18.

20. Lard LR, Visser H, Speyer I, Vander Horst-Bruinsma IE, ZWINDERMAN AH, BREEDVELD FC ET AL. Early versus delayed treatment in patients with recent-onset rheumatoid arthritis: comparison of two cohorts who received different treatment strategies. Am J Med 2001; 111: 446-51.

21. Egsmose C, Lund B, Borg G, Pettersson H, Berg E, Brodin U ET aL. Patients with rheumatoid arthritis benefit from early $2^{\text {nd }}$ line therapy: 5 year followup of a prospective double blind placebo controlled study. J Rheumatol 1995; 22: 2208-13.

22. EMERY P. Therapeutic approaches for early rheumatoid arthritis. How early? How aggressive? Br J Rheumatol 1995; 34 Suppl 2: 87-90.

23. O’DEL JR. Treating meumatoid arthritis early: a window of opportunity? Arthritis Rheum 2002; 46: 283-5.

24. Pincus T, Sokka T, Kautiainen H. Patients seen for standard rheumatoid arthritis care have significantly better articular, radiographic, laboratory, and functional status in 2000 than in 1985. Arthritis Rheum 2005; 52: 1009-19.

25. Emery P, Breedveld FC, Dougados M, Kalden JR, Schiff MH, SMOLEN JS. Early referral recommendation for newly diagnosed rheumatoid arthritis: evidence based development of a clinical guide. Ann Rheum Dis 2002; 61: 290-7.

26. Van Dongen H, Van Aken J, LaRd LR, Visser K, Ronday HK, Hulsmans HM et al. Efficacy of methotrexate treatment in patients with probable rheumatoid arthritis: a double-blind, randomized, placebo-controlled trial. Arthritis Rheum 2007; 56: 1424-32.

27. Young A. Early rheumatoid arthritis. Rheum Dis Clin North Am 2005; 31: 659-79.

28. Mitchell KL, PISETSKY DS. Early rheumatoid arthritis. Curr Opin Rheumatol 2007; 19: 278-83.

29. Kiareskog L, Stolt P, Lundberg K, Kallberg $H$, Bengtsson C, Grunewald J et al. A new model for an 
etiology of rheumatoid arthritis: smoking may trigger HLA-DR (shared epitope)-restricted immune reactions to autoantigens modified by citrullination. Arthritis Rheum 2006; 54: 38-46.

30. Massardo L, Gareca N, Cartes MA, Cervilia V, GonzáIEZ A, JACOBEL S. The presence of the HLA-DRB1 shared epitope correlates with erosive disease in Chilean patients with rheumatoid arthritis. Rheumatology (Oxford) 2002; 41: 153-6.

31. Keen HI, Brown AK, Wakefield RJ, Conaghan PG. MRI and musculoskeletal ultrasonography as diagnostic tools in early arthritis. Rheum Dis Clin North Am 2005; 31: 699-714.

32. Vargas J, Ortega C, Burdiles A, Contreras O, MassarDo L. Detection of bone erosion in rheumatoid arthritis wrist and metacarpophalangeal joints: a comparison of ultrasonography, computed tomography and magnetic resonance imaging. Arthritis Rheum 2006; 54: S134.

33. Combe B, Landewe R, Lukas C, Bolosiu HD, Breedveid F, Dougados M ET AL. EULAR recommendations for the management of early arthritis: report of a task force of the European Standing Committee for International Clinical Studies Including Therapeutics (ESCISIT). Ann Rheum Dis 2007; 66: 34-45.

34. Prevoo ML, Van T Hof MA, Kuper HH, Van Leeuwen MA, Van De Putte LB, Van Riel PL. Modified disease activity scores that include twenty-eight-joint counts. Development and validation in a prospective longitudinal study of patients with rheumatoid arthritis. Arthritis Rheum 1995; 38: 44-8.

35. Fries JF, Spitz P, Kraines RG, Holman HR. Measurement of patient outcome in arthritis. Arthritis Rheum 1980; 23: 137-45.

36. Sociedad Chilena de Reumatología. ed. Guías de tratamiento de la Artritis Reumatoide. Vol. 1. Santiago; 2004.

37. Guidelines for the management of rheumatoid arthritis: 2002 Update. Arthritis Rheum 2002; 46: 328-46.

38. Kavanaugh A, Heudebert G, Cush J, Jain R. Cost evaluation of novel therapeutics in rheumatoid arthritis (CENTRA): a decision analysis model. Semin Arthritis Rheum 1996; 25: 297-307.
39. SoKKa T, Hannonen P, MotTonen T. Conventional disease-modifying antirheumatic drugs in early arthritis. Rheum Dis Clin North Am 2005; 31: 729-44.

40. Labarca C, Massardo L, Garcia PI, Jacobew S. [Effectiveness of infliximab in patients with inflammatory arthritis refractory to conventional treatment]. Rev Med Chil 2003; 131: 1157-64.

41. Goekoop-Ruiterman YP, De Vries-Bouwstra JK, Aliaart CF, Van Zeben D, Kerstens PJ, Hazes JM et al. Clinical and radiographic outcomes of four different treatment strategies in patients with early rheumatoid arthritis (the BeSt study): a randomized, controlled trial. Arthritis Rheum 2005; 52: 3381-90.

42. Tarner IH, Muler-Ladner U, Gay S. Emerging targets of biologic therapies for rheumatoid arthritis. Nat Clin Pract Rheumatol 2007; 3: 336-45.

43. Kavanaugh A, Cohen S, Cush J. The evolving use of TNF inhibitors in rheumatoid arthritis. J Rheumatol 2004; 31: 1881-4.

44. ScotT DL, KingSLEY GH. Tumor necrosis factor inhibitors for rheumatoid arthritis. N Engl J Med 2006; 355: 704-12.

45. Feison DT, Anderson JJ, Boers M, Bombardier C, Furst D, Goldsmith C ET AL. American College of Rheumatology. Preliminary definition of improvement in rheumatoid arthritis. Arthritis Rheum 1995; 38: 72735.

46. Chen Y-F, Jobanputra P, Barton P, Jowet S, Bryan S, CLARK W, ET AL. A systematic review of the effectiveness of adalimumab, etanercept, and infliximab for the treatment of rheumatoid arthritis in adults and an economic evaluation of their cost-effectiveness. Health Technol Assess 2006; 10: 1-229.

47. Da Silva JA, Jacobs JW, Kirwan JR, Boers M, Saag KG, INES LB ET AL Safety of low dose glucocorticoid treatment in rheumatoid arthritis: published evidence and prospective trial data. Ann Rheum Dis 2006; 65: 285-93.

48. Jacobs JW, Van Everdingen AA, Verstappen SM, Bijlsma JW. Followup radiographic data on patients with rheumatoid arthritis who participated in a two-year trial of prednisone therapy or placebo. Arthritis Rheum 2006; 54: 1422-8. 